\title{
ARTICLE \\ Simulated Curved Firing Electromagnetic Gun
}

\section{Jiuxin Gong* Yuechang Shi Mingwei Xu}

School of Information Engineering, North China University of Science and Technology, Tangshan, Hebei, 063210, China

\begin{tabular}{l}
\hline ARTICLE INFO \\
\hline Article history \\
Received: 17 January 2020 \\
Revised: 24 January 2020 \\
Accepted: 9 April 2020 \\
Published Online: 16 April 2020 \\
\hline
\end{tabular}

Keywords:

Electromagnetic cannon

OpenMV4

STM32F 407

Servo head

Laser radar.

\begin{abstract}
The device uses a self-made electromagnetic cannon and a control charging and discharging circuit to constitute the launching device of the electromagnetic cannon. The two-degree-of-freedom control PTZ controls the angle and direction of the electromagnetic cannon. It uses dual power supplies to power the main control board and electromagnetic cannon device. The microprocessor STM32F407 is used as the control core of the electromagnetic cannon, and the TCA8418 IIC matrix keyboard is used to adjust the distance and angle. The OpenMV4 automatically searches for the guide mark and captures the image shape and color blocks in real time to control the electromagnetic cannon to hit the round bullseye. . In addition, it also uses Lidar to measure the distance between the calibration point and the ring target, and uses OpenMV4 to realize the automatic search and launch of the electromagnetic cannon.
\end{abstract}

magnetic force to hit the target ring target. At the same time, the relevant parameters are displayed on the LCD display.

The general block diagram of the system is shown in the figure:

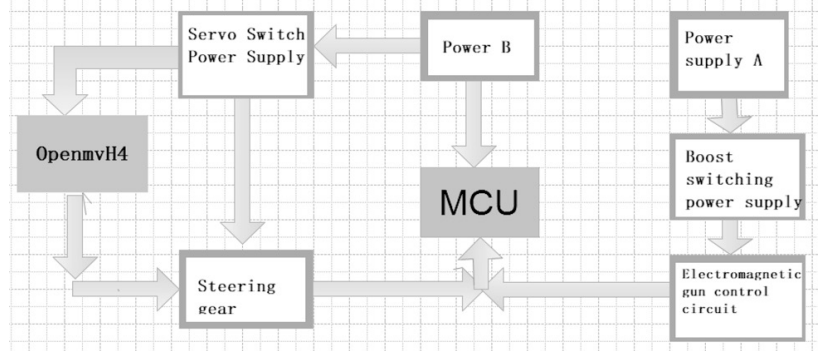

Figure 1. general block diagram of the system

*Corresponding Author:

Jiuxin Gong,

School of Information Engineering, North China University of Science and Technology, Tangshan, Hebei, 063210, China; E-mail:930525332@qq.com. 


\subsection{Mechanical Solution}

The gimbal is used as the base, the electromagnetic cannon is used as the launching device, and the OPENMV camera is used to lock the guide mark. At the same time, the main control board and the electromagnetic gun control device are placed on the bottom plate.

\subsection{Hardware solution}

The hardware consists of the main control microcontroller STM32F407, OPENMV H4 camera, Lidar, switching power supply module, and matrix keyboard.

\subsubsection{Master}

STM32MCU is fast, small, stable, rich in resources, and affordable.

Option 1: Use STMicroelectronics' STM32 F1 microcontroller.

The STM32 F1 microcontroller clock frequency reaches $72 \mathrm{MHz}$, which is the highest performance product in its class; the basic clock frequency is $36 \mathrm{MHz}$. As a development board for beginners, the STM32F103 can meet most of our functional needs.

Option 2: Use STMicroelectronics' STM32 F4 microcontroller.

The highest operating frequency of STM32F4 is $168 \mathrm{Mhz}$, while STM32F1 is $72 \mathrm{Mhz}$; STM32F4 has ART adaptive real-time accelerator, STM32F1 requires waiting period; STM32F4's FSMC uses a 32-bit multiple AHB bus matrix, which significantly improves the access speed compared to STM32F1 bus.

Finally, from the perspective of the proficiency of the chip and the clock frequency, it was decided to use the STM32F407 microcontroller in the second scheme.

\subsubsection{Camera}

\section{Option 1: Use OPENMV4}

OpenMV camera is a compact, low power, low cost circuit board that can easily complete machine vision applications. Via high-level Python scripts instead of C / C ++.

Option 2: Digital camera MT9V032

The driving configuration chip is integrated in the drill wind camera, and the dynamic threshold can be binarized with the automatic exposure function.

The title requires the ability to automatically search for a target and bombard a red ring target. In contrast, the 0penMV camera can recognize color images, so choose option one.

(1) Power supply

Option 1: Switching Power Supply RY3834
The advantages are small size, light weight, high efficiency (generally $60 \sim 70 \%$, while linear power supply is only $30 \sim 40 \%$ ), strong self-interference resistance, wide output voltage range, modularity.

The main disadvantage is that the DC voltage obtained after rectification usually causes a voltage change of $20 \%$ to $40 \%$.

Option 2: Linear voltage regulator LM2596

The LM2596 is a switching voltage regulator for a step-down power management monolithic integrated circuit, capable of outputting a $3 \mathrm{~A}$ drive current, and has good linearity and load regulation characteristics.

Considering the small size and high efficiency of the switching power supply, the first option is selected.

(2) Steering gear

\section{Option 1: Futaba s3010 servo}

The S3010 steering gear is a full resin gear, suitable for use in primary electric / oil-powered car models, electric / oil-powered ship models, general fixed-wing and small fixed-wing models, and medium-level oil-powered / electric 30-level helicopters.

Option 2: DG 995 steering gear

Digital servos have fast response, accurate angles, high torque, double bearings, small internal resistance, nonshake rudder, stall protection function, completely get rid of the phenomenon of servo shake, stalled burnout and other phenomena.

During the debugging process, the Futaba s3010 servo was selected to find the guide mark, and the gun barrel wobbled, resulting in angular deviation. Therefore, the DG 995 digital servo with debounce function was selected in the second scheme to improve the stability.

(3) keyboard

Option 1: TCA8418 matrix keyboard

The TCA8418 is a keyboard scan device with integrated ESD protection. It has 18 GPIOs, which can support up to 80 keys through the $\mathrm{I} 2 \mathrm{C}$ interface.

Option 2: Traditional buttons

The independent button type is a single button circuit composed of I / O port lines directly. The advantage is that the work of each button will not affect the state of other I / O port lines. Although the programming is simple, the single-chip IO port is seriously wasted, and the IO resources of the single-chip microcomputer system are limited.

The TCA8418 matrix keyboard has strong operability, short debounce time, and convenient use. Considering Option 1 comprehensively.

\section{Theoretical Analysis}

\subsection{Analysis of Electromagnetic Gun Model}

The coil electromagnetic gun is mainly composed of a 
barrel, an excitation energy source, a relay, and a barrel. A single-stage or multi-stage excitation coil is closely wound on the barrel, and the excitation energy is a large-capacity capacitor. Based on the principle of electromagnetic induction, the control triggers a control switch to discharge, so that the discharge circuit is turned on, and a large instantaneous pulse of current passes through the coil, which generates a magnetic field. The transient magnetic field generated by the exciting coil causes the coil or ferromagnetic projectile to generate an induced current or magnetization, and then forms a magnetic force that attracts each other, thereby driving the coil or ferromagnetic projectile to move.

\subsection{Coil Body Stress}

The elastic body a is wound with a certain number of coils, as shown in Figure 2. When the solenoid passes a current $i$, the induced current of the elastic body coil is i. For the convenience of calculation, at the moment of interaction, it can be considered as constant. According to Ampere force and Stokes formula;

$d \vec{F}=i_{2} d l * \vec{B}$

$\oint \vec{B} \circ d \vec{l}=\iint_{S}(\nabla * \vec{B}) \circ d \vec{S}$

$\vec{F}=\oint_{l} i_{2} d \vec{l} * \vec{B}=i_{2} \nabla \varphi$

The formula for the translational force of the coil in the magnetic field can be deduced. The translational force at the center of the coil is zero, and the translational thrust at the bottom of the coil is the largest. Therefore, the cannonball is placed at the bottom when it is launched.
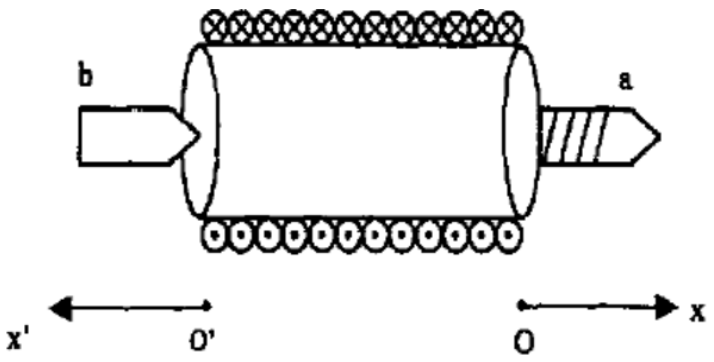

Figure 2. Force analysis

\subsection{Ballistic Kinematics Analysis}

According to Newton's second law, the differential equation of kinematics of the parabola can be obtained as: $m \frac{d x^{2}}{d t^{2}}=-\frac{1}{2} i^{2} \frac{d l_{x}}{d t}$

\subsection{Energy Calculation}

Set the ferromagnetic projectile outside the solenoid port, the distance is $x-l / 2$ (Initial position of the projectile), If $x-l / 2$ Much smaller than the solenoid length $l$, Then the magnetic field strength at the projectile can be approximated as

$H=\frac{l}{|x|+\frac{l}{2}} n i$ tile:

Magnetie flux through the cross section of the projec-

$\varphi_{m}=\frac{l}{|x|+\frac{l}{2}} \mu_{0} n i S$

According to the continuous flux theorem and the definition of inductance, the inductance of the solenoid is:

$L_{x}=\frac{n l \varphi_{M}}{i}$

The total system energy is:

$W_{m}=\int_{0}^{\phi_{m}} i d \phi_{m}=\frac{1}{2} L_{x} i^{2}$

\section{Theoretical Parameters}

\subsection{Relationship between Emission Distance and PWM}

Use the transmission distance as the independent variable and the PWM value as the dependent variable to explore the relationship. However, the magnitude of the supply voltage affects the transmission distance, so first determine the optimum value of the supply voltage.

(1) When the power supply voltage is $21.81 \mathrm{~V}$

Table 1. Distance and PWM value

\begin{tabular}{|c|c|}
\hline Distance mean & PWM value \\
\hline 200 & 4050 \\
\hline 210 & 4100 \\
\hline 223.5 & 4130 \\
\hline 252 & 4200 \\
\hline 277.5 & 4500 \\
\hline
\end{tabular}

The function fit is shown in the figure: 


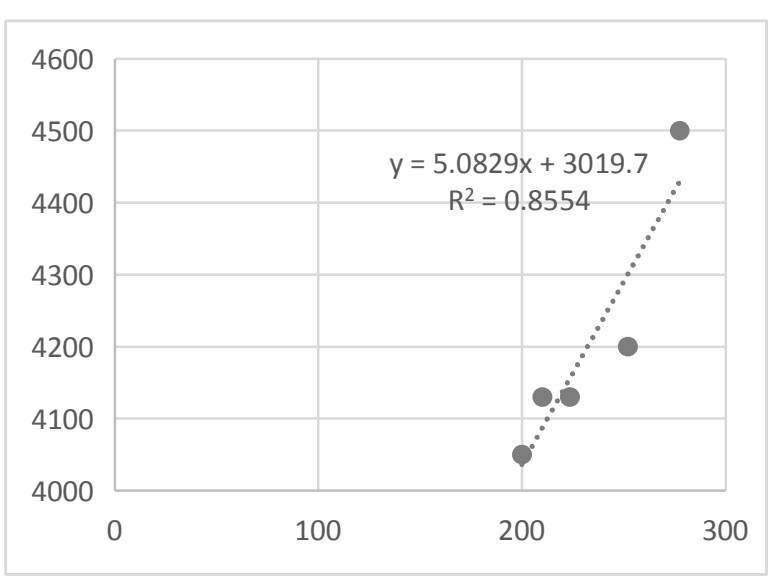

Figure 3. Relation between transmission distance and PWM

The resulting functional relationship is:

$Y=5.0829 X^{2}+3019.7$

(2) When the power supply voltage is $24.84 \mathrm{~V}$

Table 2 Distance and PWM values

\begin{tabular}{|c|c|}
\hline Distance mean & PWM value \\
\hline 225 & 3360 \\
\hline 250 & 3300 \\
\hline 266 & 3260 \\
\hline 273 & 3200 \\
\hline 296.5 & 3140 \\
\hline
\end{tabular}

The function fit is shown in the figure:

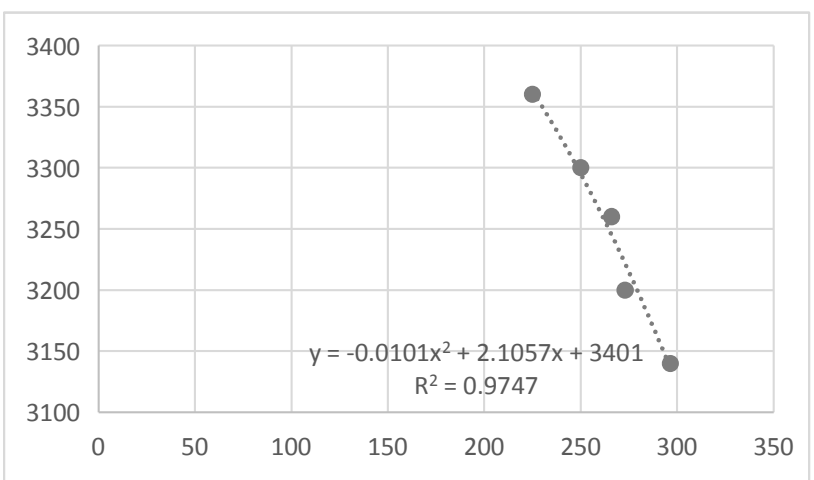

Figure 4. The relationship between the transmission distance and the PWM

The resulting functional relationship is:

$Y=-0.0101 X^{2}+2.1057 X+3401$

The experiment found that when the power supply voltage was $24.84 \mathrm{~V}$, the fitted degree of the function sought was the best and the deviation was the smallest.

\subsection{Relationship between Angle and PWM}

The title requires that the circular target is placed horizontally on the ground, and the position of the bull's eye is within the range of an angle \pm 30 degree from the center axis. For the convenience of calculation, $30^{\circ}$ left of the central axis is used as the experimental calibration $0^{\circ}$, and $30^{\circ}$ right of the central axis is used as the experimental calibration $60^{\circ}$. The following is the recorded experimental data:

Table 3. Angle and PWM values

\begin{tabular}{|c|c|}
\hline Angle $(\mathrm{X})$ & PWM value $(\mathrm{Y})$ \\
\hline 0 & 3100 \\
\hline 10 & 2950 \\
\hline 30 & 2680 \\
\hline 50 & 2420 \\
\hline
\end{tabular}

The function fit is shown in the figure:

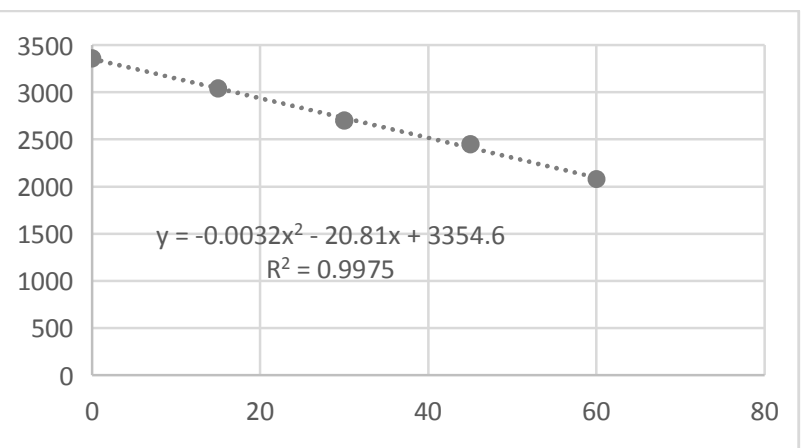

Figure 5. Relationship between angle and PWM

The resulting functional relationship is:

$Y=-0.0032 X^{2}-20.81 X+3354.6$

\section{Circuit and Programming}

\subsection{Design of the Circuit}
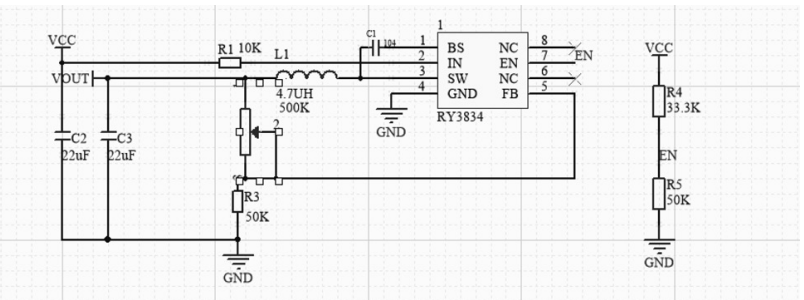

\subsection{Control Scheme}

Using PID control algorithm

Option 1: Position-type PID control. 
Positional PID is the actual position of the current system, the deviation from the expected position you want to achieve, PID control. When the position type PID reaches saturation, the error will still continue to accumulate under the action of integration. Once the error starts to change in reverse, the system needs a certain time to exit from the saturation region, so when $\mathrm{u}(\mathrm{k})$ reaches the maximum and minimum, it must stop Integral effect, and there must be integral limit and output limit.

So when using positional PID, we generally use PD control directly.

Option 2: Incremental PID control.

Incremental PID is to increment the position-type PID. At this time, the controller outputs the difference between the position values calculated at two adjacent sampling times, and the result is an increment, that is, the value of the last control amount. On the basis, the amount of control needs to be increased (negative value means decreased).

Accumulation is not required in incremental PID. The determination of the control increment $\Delta \mathrm{u}(\mathrm{k})$ is only related to the last 3 sampling values. It is easy to obtain a better control effect through weighting processing, and the incremental operation will not seriously affect the work of the system when a problem occurs in the system. To sum up, choose the second incremental PID.

\subsection{Program Design}

(1) Program Function Description

The high and low level of the Io port of the single-chip microcomputer is controlled by the program, thereby controlling the opening and closing of the relay to realize the charging and discharging of the capacitor, thereby generating an instantaneous voltage change, and the changed voltage generates a magnetic field to drive the small iron rod to be driven outward by the electromagnetic force.

(2) Partial Program Explanation Display Part

By controlling the FSMC LCD to realize the mode, angle and distance display, it is more intuitive to see the change of variables. Keyboard part: TCA8418 matrix keyboard adopts analog IIC communication, and uses a minimum number of IO ports to control more keys, which greatly saves the resources of the microcontroller. Laser ranging: The target distance is transmitted to the single-chip microcomputer by serial communication.

\section{System Test Results}

\subsection{Xperimental Design}

Design the circuit diagram. Use an enameled wire with a diameter of $1.2 \mathrm{~mm}$ to wind on a plastic straw with an inner diameter of about $1 \mathrm{~cm}$ and a length of $18 \mathrm{~cm}$. The enameled wire is a $5 \mathrm{~cm}$ long solenoid. It is powered by $24 \mathrm{~V} \mathrm{DC}$, and a $5 \mathrm{~V}$ DC relay is used to control the charge and discharge of an electrolytic capacitor. The bullet body is an ordinary nail head. Put the projectile into the bottom of the solenoid during the experiment.

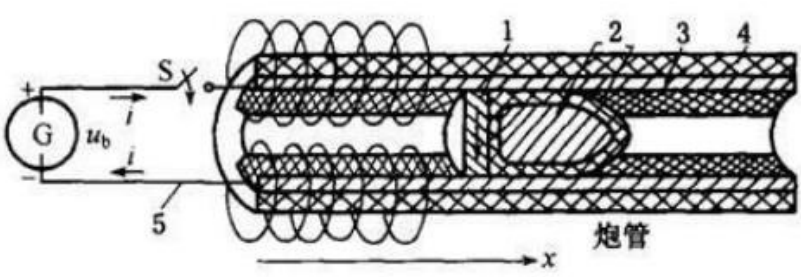

Figure 6. Simulation experiment circuit diagram

\subsection{Parameter Measurement}

\subsubsection{Actual Distance Value}

When the supply voltage is $24.84 \mathrm{~V}$, the relationship between the transmission distance and the PWM is:

$Y=-0.0101 X^{2}+2.1057 X+3401$

Calculate the PWM value according to the formula, input the $\mathrm{d}$ value with different distance from the matrix keyboard, and the electromagnetic cannon launches the projectile to different positions of the target. Then actually measure the distance value and calculate the distance error at the same time, as shown in the following table:

Table 4. Measured distance

\begin{tabular}{|c|c|c|c|}
\hline $\begin{array}{c}\text { Distance stan- } \\
\text { dard value }(\mathrm{cm})\end{array}$ & $\begin{array}{c}\text { PWM } \\
\text { calculation }\end{array}$ & $\begin{array}{c}\text { Distance actual value } \\
(\mathrm{cm})\end{array}$ & Distance error \\
\hline 210 & 3398 & 210 & 0 \\
\hline 230 & 3351 & 231 & 0.0043 \\
\hline 255 & 3281 & 256 & 0.0039 \\
\hline 270 & 3233 & 272 & 0.0074 \\
\hline 290 & 3162 & 291.5 & 0.0052 \\
\hline
\end{tabular}

From the table, it can be analyzed that the distance error is small and meets the requirement that the absolute value of the distance deviation is less than $50 \mathrm{~cm}$, indicating that the system can complete the basic requirements (2).

\subsubsection{Relationship between Angle and PWM}

The title requires that the circular target is placed hori- 
zontally on the ground, and the position of the bull's eye is within the range of an angle a?30 ?from the center axis. For the convenience of calculation, 30 ?left of the central axis is used as the experimental calibration 0 ? and 30 ?right of the central axis is used as the experimental calibration 60 ?

The relationship between angle and PWM is:

$Y=-0.0032 X^{2}-20.81 X+3354.6$

Calculate the PWM value according to the formula. Use the keyboard to input the distance $d$ between the center of the ring target and the calibration point and the deviation angle $\alpha$ from the center axis to the electromagnetic gun. The actual measurement results in an angle value, and the angle error is calculated at the same time. The following is the recorded experimental data:

Table 5. Measured angle

\begin{tabular}{|c|c|c|c|}
\hline $\begin{array}{c}\text { Standard value of } \\
\text { angle (degrees) }\end{array}$ & PWM value & Actual angle (degrees) & error \\
\hline 0 & 3100 & 2 & 0.07 \\
\hline 10 & 2950 & 11 & 0.1 \\
\hline 30 & 2680 & 32 & 0.07 \\
\hline 50 & 2420 & 49 & 0.02 \\
\hline
\end{tabular}

From the table, it can be analyzed that the distance error is small, and the measured distance is combined with the actual value. It meets the requirements for automatic aiming and shooting of the electromagnetic gun after onekey startup, indicating that the system can fulfill the basic requirements (3).

\subsubsection{Play part}

Part of the task is combined with the OpenMV4 camera and the relationship between distance, angle and PWM, which can achieve the positioning of distance and angle.

\section{Summary and Perception}

The construction of the mechanical structure is very important. The structure is constantly adjusted and the chip is replaced. There were many problems in the middle, but they were finally resolved. From writing the code to the final success display and completing the test, I have experienced countless debugging and improvements in the middle. Fail, think, discuss, adapt, test, and solve the unexpected difficulties you face again and again. It is also a challenge for us, but persistence is our unanimous belief. Now that you can choose a challenge, you must do it with all your heart. At the same time, communicate with your own team members and other team members. This will not only promote the learning of our team members, but also find and deal with problems in time.

\section{References}

[1] Liu Huoliang and Yang Sen, STM32 Library Development Practical Guide, Beijing: Machinery Industry Press, 2008.

[2] Tan Haoqiang. C language programming [M]. Beijing: Tsinghua University Press, 2012.

[3] Huang Ying. Two self-made controllable electromagnetic gun models are used to demonstrate the law of electromagnetic induction in university physics. [D]. Journal of Central China Normal University (Natural Science Edition). 2012. (01): 89-91.

[4] Hu Dezhi, Li Haijun, Wang Yuxin, et al. Design and production of three-stage coaxial coil electromagnetic gun model [D] .Journal of North China Institute of Science and Technology, 2010,7 (1): 85 -87. 